\title{
Stage IB Bone Sarcoma AJCC v7
}

National Cancer Institute

\section{Source}

National Cancer Institute. Stage IB Bone Sarcoma A/CC V7. NCI Thesaurus. Code C6465.

Stage IB includes: (T2, N0, M0, G1, G2, GX); (T3, N0, M0, G1, G2, GX). T2: Tumor more than $8 \mathrm{~cm}$ in greatest dimension. T3: Discontinuous tumors in the primary bone site. $\mathrm{NO}$ : No regional lymph node metastasis. M0: No distant metastasis. G1: Well differentiatedlow grade. G2: Moderately differentiated-low grade. GX: Grade cannot be assessed. (AJCC 7th ed.) 